\title{
Microbiome: from community metabolism to host diseases
}

\author{
Nan Qin $^{1,2^{*}}$, Xiuzhu Dong ${ }^{3^{*}}$ \& Liping Zhao ${ }^{4,5^{*}}$ \\ ${ }^{1}$ Shanghai Tenth People's Hospital Affiliated to Tongji University, Shanghai 200072, China; \\ ${ }^{2}$ Realbio Genomics Institute, Shanghai 200050, China; \\ ${ }^{3}$ State Key Laboratory of Microbial Resources, Institute of Microbiology, Chinese Academy of Sciences, Beijing 100101, China; \\ ${ }^{4}$ State Key Laboratory of Microbial Metabolism and Ministry of Education Key Laboratory of Systems Biomedicine, School of Life Sciences and \\ Biotechnology, Shanghai Jiao Tong University, Shanghai 200240, China; \\ ${ }^{5}$ Department of Biochemistry and Microbiology and New Jersey Institute for Food, Nutrition, and Health, School of Environmental and \\ Biological Sciences, Rutgers University, New Brunswick, NJ 08901, USA
}

Received June 14, 2018; published online June 22, 2018

Citation: Qin, N., Dong, X., and Zhao, L. (2018). Microbiome: from community metabolism to host diseases. Sci China Life Sci 61, 741-743. https://doi.org/ $10.1007 / \mathrm{s} 11427-018-9335-8$

Microorganisms live in almost every habitat on the earth from the poles to the equator, from forest to desert, from mountain to deep sea, from geysers to karst structures, as well as the body of almost all multicellular organisms from plants to animals. Essentially, species among the microbial populations interact in different ways, as predators and prey, or as symbionts in the form of communities, so adapt to hosts and environments through nutrient and energy exchange. This issue of Science China Life Sciences includes two reviews and four research articles, which investigate the roles of microbial communities in maintaining ecological balances and explore the possible mechanisms of their interactions with hosts and environments.

\section{HUMAN MICROBIOTA AND DISEASES}

The human microbiota refers to the aggregate of commensal, symbiotic and pathogenic microorganisms found in and on the human body, and predominantly, in the gut (Peterson et al., 2009). Microbiota has been demonstrated to be crucial in the immunological, hormonal and metabolic homeostasis of the host, and play critical roles in the development of a

\footnotetext{
*Corresponding authors (Nan Qin, email: qinnan001@126.com; Xiuzhu Dong, email:
} dongxz@im.ac.cn; Liping Zhao, email: lpzhao@sjtu.edu.cn) variety of diseases, including obesity, type 2 diabetes, immune-mediated inflammatory diseases, and even cardiovascular and mental problems (Quigley, 2013). In the last two decades, remarkable advances in the sequencing technologies and bioinformatics have greatly helped the exploration of the human microbiome, which describes the collective genomes of the human microbiota their metabolic activities, and the corresponding effects to human health (Weinstock, 2012).

Chronic functional constipation is a kind of common intestinal condition that occurs in children, adults, and elderly people. Huang et al. summarize recent studies which have focused on the connection between constipation and intestinal flora disturbance. A theoretical basis for microbial treatment of chronic constipation is provided based on the findings that particular metabolites produced by gut bacteria such as short chain fatty acids and 5-HT are of great importance in maintaining bowel regularity and avoiding intestinal dysfunction and difficulty in emptying. The safety, convenience and curative effect of microbial treatment including probiotic preparations (i.e. probiotics, prebiotics, synbiotics) and fecal microbiota transplantation (FMT) were discussed thoroughly (Huang et al., 2018). Interestingly, Zhang et al. also report the long-term effect of FMT combined with soluble dietary fiber (pectin) in treating slow transit constipation (STC). They found that patients receiv- 
ing FMT therapy for three months and soluble dietary fiber daily for one year displayed obvious improvements in spontaneous bowel movements, stool consistency and the Wexner constipation scale, which indicate the great clinical potential of FMT in remit constipation (Zhang et al., 2018).

Non-alcoholic fatty liver disease (NAFLD) is a chronic liver disease which results from local fat deposition. The disease has been shown to be tightly associated with the dysbiosis of gut microbiota. Prevalence of NAFLD is increasing in an unexpected speed in China and has reached $20.9 \%$ of total population, which may lead to severe public health problems ( $\mathrm{Li}$ et al., 2014). Li et al. introduce their study on profiling the gut microbiota structure of NAFLD patients. Compositional differences in fecal bacterial communities of NAFLD patients compared with healthy controls were revealed on family and genus levels. Furthermore, insulin resistance, waist-to-hip ratio (WHR) and body mass index (BMI) were shown to be closely related to bacterial community structure changes and development of NAFLD (Li et al., 2018).

Mouse models have been widely employed to decipher the underlying mechanisms of human diseases as well as to explore and evaluate novel therapeutic agents and drugs (McGonigle and Ruggeri, 2014). Li et al. have examined the influence of initial gut microbiota on toxics-induced colitis mouse models. Significant variations in the initial gut microbiota of different batches of mice were found. Composition of the gut microbiota in mice with severe colitis, as compared with mice with milder colon damage, had unique features, such as an increase in Akkermansia bacteria and a decrease in Barnesiella spp. Moreover, these varied gut bacteria in the different trials also showed different responses to DSS treatment. They suggest that, in studies using mouse models, the gut microbiota must be considered when examining mechanisms of diseases to ensure comparable results (Li et al., 2018).

\section{METABOLIC INTERACTIONS IN MICROBIAL COMMUNITIES}

Microbes are intermingled with each other through complex metabolic links. Exploration of microbial metabolic interactions in the community context, as opposed to studying bacterial metabolism in pure culture, can help with deep understanding of interactions within the community members, reveal new phenotypes, help designing synthetic communities for biotechnology, and enable cultivating the "uncultivables" (Ponomarova and Patil, 2015).

In this issue, Zheng et al. investigate the microbe-inherent electron transfer process by a direct interspecies electron transfer (DIET) model between Geobacter metallireducens and Geobacter sulfurreducens in the bicultures (Zheng et al.,
2018). In this model, the two Geobacter species could achieve ethanol oxidation by transferring the electrons from ethanol to fumarate. The findings suggest the importance of comparative transcriptomics as a promising route to reveal different responses of electron donor and acceptor during DIET in co-cultures, and to expand understanding of bacterial interactions in natural microbial communities that underpins syntrophic metabolism within a microbiome.

Nitrogen pollution is an increasingly severe worldwide problem, while drainage of nitrogen-containing wastewater has been one of the most important cause. In a review article, Miao and Liu summarize the recent advances in microbiome and-omics studies on the microbial denitrification processes in wastewater treatment. Powered by the "meta-omics" technologies including genomics, transcriptomics, proteomics and metabolomics, researchers have gained great insight into the microbial community structure, key functional microbes and their physiology, functional genes involved in nitrogen cycle, and responses of microbes and their genes to changes of environmental factors or operating parameters, in denitrification processes in wastewater treatment systems (Miao and Liu 2018).

All these research articles and reviews highlight the importance of integrated microbiome and -omics approaches which have great future for more in-depth, more systematic understanding of interactions between hosts and microbes, between environmental parameters and microbes, as well as among different microbial members. Taking advantages of these rapidly developing technologies, more work is in need to open up new horizons in our understanding of the vast microbial "dark matter".

Compliance and ethics The author(s) declare that they have no conflict of interest.

Huang, L., Zhu, Q., Qu, X., and Qin, H. (2018). Microbial treatment in chronic constipation. Sci China Life Sci 61, 744-752.

Li, F., Sun, G., Wang, Z., Wu, W., Guo, H., Peng, L., Wu, L., Guo, X., and Yang, Y. (2018). Characteristics of fecal microbiota in non-alcoholic fatty liver disease patients. Sci China Life Sci 61, 770-778.

Li, M., Wu, Y., Hu, Y., Zhao, L., and Zhang, C. (2017). Initial gut microbiota structure affects sensitivity to DSS-induced colitis in a mouse model. Sci China Life Sci 61, 762-769.

Li, Z.Z., Xue, J., Chen, P., Chen, L.Z., Yan, S.P., and Liu, L.Y. (2014). Prevalence of nonalcoholic fatty liver disease in mainland of China: a meta-analysis of published studies. J Gastroenterol Hepatol 29, 42-51.

McGonigle, P., and Ruggeri, B. (2014). Animal models of human disease: challenges in enabling translation. Biochem Pharmacol 87, 162-171.

Miao, L., and Liu, Z. (2018). Microbiome analysis and -omics studies of microbial denitrification processes in wastewater treatment: recent advances. Sci China Life Sci 61, 753-761.

Peterson, J., Garges, S., Giovanni, M., McInnes, P., Wang, L., Schloss, J.A., Bonazzi, V., McEwen, J.E., Wetterstrand, K.A., Deal, C., et al. (2009). The NIH Human Microbiome Project. Genome Res 19, 2317-2323.

Ponomarova, O., and Patil, K.R. (2015). Metabolic interactions in microbial communities: untangling the Gordian knot. Curr Opin Microbiol 27, 37-44.

Quigley, E.M. (2013). Gut bacteria in health and disease. Gastroenterol 
Hepatol (N Y) 9, 560-569.

Weinstock, G.M. (2012). Genomic approaches to studying the human microbiota. Nature 489, 250-256.

Zhang, X., Tian, H., Gu, L., Nie, Y., Ding, C., Ge, X., Yang, B., Gong, J., and $\mathrm{Li}, \mathrm{N}$. (2018). Long-term follow-up of the effects of fecal microbiota transplantation in combination with soluble dietary fiber as a th- erapeutic regimen in slow transit constipation. Sci China Life Sci 61, 779-786.

Zheng, S., Liu, F., Li, M., Xiao, L., and Wang, O. (2018). Comparative transcriptomic insights into the mechanisms of electron transfer in Geobacter co-cultures with activated carbon and magnetite. Sci China Life Sci 61, 787-798.

\section{Biographical Sketch}

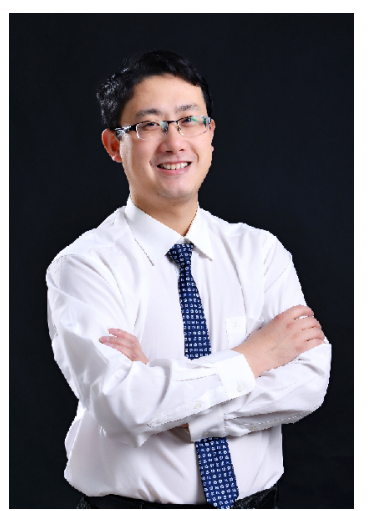

Dr. Nan Qin is a professor of Shanghai Tenth People's Hospital Affiliated to Tongji University and the founder of Shanghai Realbio Technology. He received his Ph.D. from the Department of Biological Sciences, Virginia Tech, USA in 2008. Afterwards, he joined BGI in Shenzhen as the director of department of microbial genomics. In 2011, he joined Zhejiang University School of Medicine as an associated professor and doctoral supervisor in Collaborative Innovation Center of Diagnosis and Treatment of Infectious Diseases. He won the title of "Shanghai One Thousand Talents Scheme" in 2017 and was recognized as one of Grand Challenges 2015-Young Scientists by Ministry of Science and Technology and Bill \& Melinda Gates Foundation. Dr. Qin is specialized in gut microbiome in infectious diseases, metabolic diseases, autoimmune diseases and cancer immunotherapy and he has published more than 30 papers in Nature, Science, PLoS Biol and other top academic journals since 2009 .

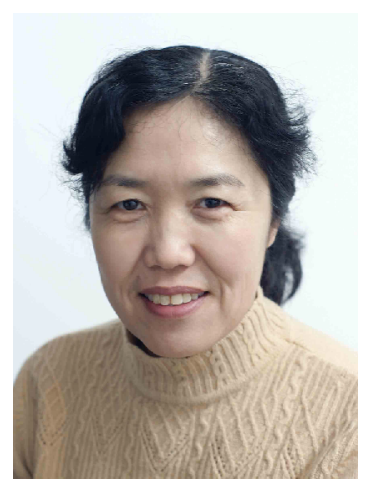

Dr. Xiuzhu Dong is a professor of Institute of Microbiology, Chinese Academy of Sciences (CAS). She received her Ph.D. degree from the Department of Microbiology, Wageningen University of the Netherlands in 1994, and did her postdoctoral research in University of Alabama at Birmingham, USA from 1995 to 1996. Since then Dr. Dong has set up her research laboratory of anaerobes in the Institute of Microbiology, CAS. She was the director of State Key Laboratory of Microbial Resources from 2008 to 2017, and became the deputy director general of the institute in 2011. Dr. Dong was awarded by the National Science Fund for Distinguished Young Scholars in 2000 and elected to the One Hundred Talents Program of CAS in 2014. She held the chief scientist of the National Basic Research Program of China (973 Program) granted by Ministry of Science and Technology of China from 2004 to 2009. Dr. Dong is engaged in anaerobic bacteria and archaea that function in anaerobic degradation of organics to produce methane, and specialized in their physiology and antioxidative mechanisms. She has published more than 90 papers in Nucleic Acids Res, ISME J, Mol Microbiol, Environ Microbiol, Appl Environ Microbiol J Bacterial.

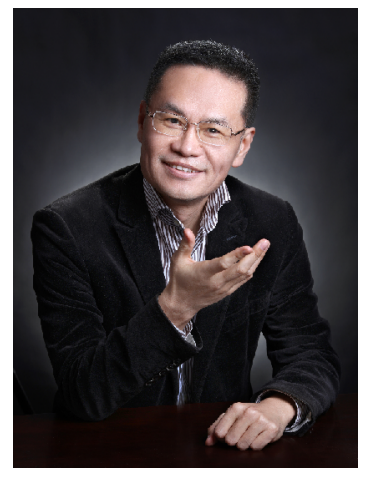

Liping Zhao is currently the Eveleigh-Fenton Chair of Applied Microbiology at Rutgers University and Distinguished Professor of Microbiology at Shanghai Jiao Tong University. He is a senior editor of the ISME $J$ and associate editor of the journal Microbiome. He is a fellow of American Academy of Microbiology. He is a senior fellow of Canadian Institute for Advanced Research (CIFAR). He serves on Scientific Advisory Board for AGA Center for Microbiome Research and Education. His team has pioneered the approach of applying metagenomics-metabolomics integrated tools and dietary intervention for systems understanding and predictive manipulation of gut microbiota to improve human metabolic health. Following the logic of Koch's postulates, Liping has found that an endotoxin-producing opportunistic pathogen isolated from an obese human gut can induce obesity in germfree mice. Their clinical trials published in Science and EBioMedicine showed that dietary modulation of gut microbiota can significantly alleviate metabolic diseases including a genetic form of obesity in children and type 2 diabetes in adults. The Science magazine featured a story on how he combines traditional Chinese medicine and gut microbiota study to understand and fight obesity (Science 336: 1248). 